\title{
Beobachtungen beschreiben
}

\author{
WISSENSCHAFT ERKLÄRT: DESKRIPTIVE STUDIEN Studiendesigns lassen sich in drei \\ große Gruppen einteilen: Deskriptive, analytische und experimentelle Studien. Jan \\ Mehrholz erläutert hier, was deskriptive Studien sind und was sie beschreiben können.
}

\section{D}

eskriptive Studien sind beobachtende oder beschreibende Studien. Sie bilden die unterste Stufe der wissenschaftlichen Hierarchie und sind oft der erste „Streifzug” im Erkenntnisprozess beispielsweise einer neuen Krankheit. Forscher unternehmen deskriptive Studien, um die Häufigkeit, den natürlichen Verlauf und mögliche Determinanten einer Krankheit zu beschreiben [1]. Aus den Ergebnissen können sie Hypothesen ableiten, welche sie dann wiederum einer rigoroseren Forschung unterziehen können, beispielsweise in analytischen oder experimentellen Studien.

Zu Beginn steht der Einzelfall $>$ Zu den deskriptiven Studien gehören Fallberichte und Fallserien, ökologische Studien und Prävalenzstudien [2]. Beobachten Forscher ungewöhnliche oder neue Befunde an Patienten, bildet dies meist den Ausgangspunkt für Fallberichte. Das ist dann der erste Schritt, unbekannte Krankheiten oder neue Therapien zu identifizieren, zu beschreiben oder zu erforschen. Da Fallberichte lediglich einzelne Patienten beobachten, kann man deren Ergebnisse nicht problemlos verallgemeinern [1].

\section{Ökologische Studien stellen Hypothesen} auf > Im Gegensatz dazu beschreibt eine ökologische Studie - auch Korrelationsstudie genannt - Krankheitshäufigkeiten im Zusammenhang mit bestimmten Faktoren [2]. Die Vorteile dieser Studie liegen auf der Hand: Sie ermöglicht einen schnellen und kostengünsti-

\section{ZUM WEITERLESEN}

Lesen Sie in der nächsten Ausgabe von ergopraxis die Fortsetzung der großen Studientypen: Analytische und experimentelle Studien!
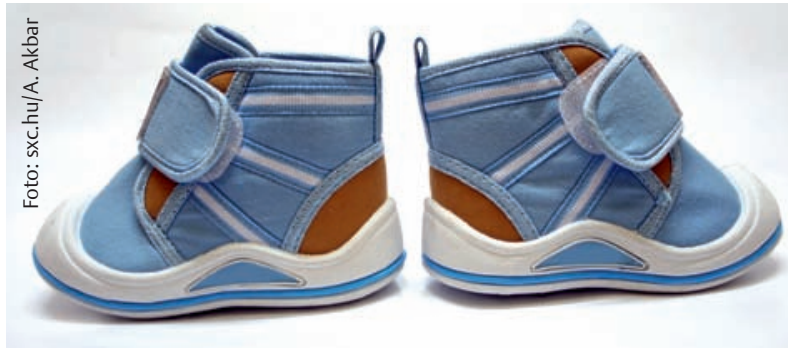

Auch in der Forschung muss man klein anfangen und erste Schritte beispielsweise mit einem Fallbericht machen. gen Zugang zu Daten und erzeugt Hypothesen. Allerdings lassen sich kaum kausale Rückschlüsse ziehen, und es tritt nicht selten ein „ökologischer Trugschluss" auf [3]. Die durch die koronare Herzkrankheit verursachte Mortalitätsrate korreliert beispielsweise mit der Anzahl von Farbfernsehern in der Bevölkerung [2]. Man kann jedoch kaum gegen den Verkauf von Fernsehgeräten zur Prävention von Herz-Kreislauf-Erkrankungen argumentieren. Erstens ist die Plausibilität zu gering, zweitens bestehen weitere Risikofaktoren und drittens kann man die Kausalität eines solchen Zusammenhangs nur bedingt nachweisen.

Prävalenzstudien, Querschnittstudien und Surveys sind Synonyme > Ein großer Teil des Wissens über Morbidität und Mortalität stammt aus Prävalenzstudien - auch Querschnittstudien oder Surveys genannt. Sie bestimmen und beschreiben den Gesundheitszustand, Krankheiten, Risikofaktoren, bestimmte Verhaltensweisen und wie eine Zielgruppe Präventionsprogramme nutzt [4]. Prävalenzstudien bieten viele Vorteile. Sie sind relativ einfach durchführbar, zum Beispiel anhand eines Fragebogens, ethisch vertretbar und im Vergleich zu anderen Studienformen kostengünstig, da sie keine Nachkontrollen erfordern. Allerdings besteht oft keine Möglichkeit, die Kausalität zu bestimmen [5].
Surveys liefern wichtige Daten > Querschnittstudien sind in den USA sehr beliebt. Forscher erheben zum Beispiel durch periodische Umfragen in regelmäßigen Abständen systematisch die Gesundheit in bestimmten Bundesstaaten. Dabei müssen sie nicht immer dieselben Personen untersuchen. Ein weiteres Beispiel für periodische Surveys ist das Thyroid-Krebs-Screening im Anschlusszeitraum an das Reaktorunglück von Tschernobyl 1986 [6]. Prävalenzstudien sind auch in der Arzneimittelforschung angezeigt und von elementarer gesundheitswissenschaftlicher Bedeutung. Anhand ihrer Daten kann man unerwünschte und unerwartete ArzneimittelNebenwirkungen beobachten. Jan Mehrholz

\section{$\Rightarrow$ Das Literaturverzeichnis finden Sie unter www.thieme.de/ergoonline > "Artikel“ > „Wissenschaft erklärt“.}

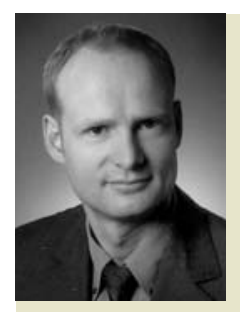

Prof. Dr. Jan Mehrholz, derzeit Gastprofessor an der California State University, leitet das Wissenschaftliche Institut der Privaten Europäischen Medizinischen Akademie in Kreischa und ist in Gera Professor für Therapiewissenschaften. 